Working

Paper

Department

of Economics

$\mathrm{Ca}^{\prime}$ Foscari University of

Venice

Guido Cazzavillan

Patrick Pintus

Endogenous Business Cycles and dynamic Inefficiency 


\title{
Endogenous Business Cycles and Dynamic Inefficiency
}

\author{
Guido Cazzavillan \\ University of $V$ enice and SET, Italy
}

\section{Patrick Pintus}

Université de la Méditerranée and GREQAM-IDEP, France

\begin{abstract}
This paper explores how the occurrence of local indeterminacy and endogenous business cycles relates to dynamic inefficiency, as defined by Malinvaud (1953), Phelps (1965) and Cass (1972). We follow Reichlin (1986) and Grandmont (1993) by considering a two-period OLG model of capital accumulation with labor-leisure choice into the first-period of agents' life and consumption in both periods. We first show that local indeterminacy and Hopf bifurcation are necessarily associated with a capital-labor ratio that is, at steady state, larger than the Golden Rule level. Consequently, paths converging asymptotically towards the steady state are shown to be dynamically inefficient, as there always exists another trajectory that starts with the same initial conditions and produces more aggregate consumption at all future dates. More surprising, however, is our main result showing that stable orbits, generated around a dynamically inefficient steady state through a supercritical Hopf bifurcation, may, in contrast, be dynamically efficient.
\end{abstract}

\section{Keywords}

Overlapping generations, endogenous labor supply, multiple equilibria, endogenous fluctuations, dynamic inefficiency.

\section{JEL Codes}

C62, D61, E32.

\author{
Address for correspondence: \\ Guido Cazzavillan \\ Department of Economics \\ Ca' Foscari University of Venice \\ Cannaregio 873, Fondamenta S.Giobbe \\ 30121 Venezia - Italy \\ Phone: (++39) 0412349152 \\ Fax: (++39) 0412349176 \\ e-mail: guido@unive.it
}

This Working Paper is published under the auspices of the Department of Economics of the Ca' Foscari University of Venice. Opinions expressed herein are those of the authors and not those of the Department. The Working Paper series is designed to divulge preliminary or incomplete work, circulated to favour discussion and comments. Citation of this paper should consider its provisional character.

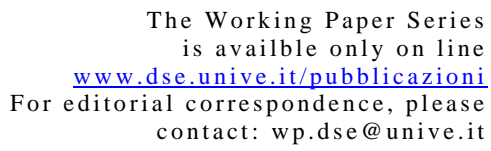

The Working Paper Series

is availble only on line www.dse.unive.it/pubblicazioni For editorial correspondence, please contact:wp.dse@unive.it

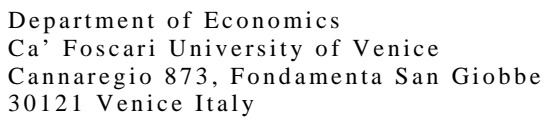

Department of Economics

Ca' Foscari University of Venice

Cannaregio 873, Fondamenta San Giobbe

30121 Venice Italy

Fax: ++390412349210 


\section{INTRODUCTION ${ }^{1}$}

This paper explores how the occurrence of local indeterminacy and endogenous business cycles relates to dynamic inefficiency, as defined by Malinvaud (1953), Phelps (1965) and Cass (1972). We follow Reichlin (1986) and Grandmont (1993) by considering a two-period OLG model of capital accumulation with labor-leisure choice into the first-period of agents' life and consumption in both periods (see also the studies of related models by, among the others, Woodford, 1984, Geanakoplos and Polemarchakis, 1986, Benhabib and Laroque, 1988, d'Aspremont, Dos Santos Ferreira and GérardVaret, 1995, Lloyd-Braga, 1995, de Vilder, 1996, Cazzavillan and Pintus, 2004, Nourry and Venditti, 2006). We show that local indeterminacy and Hopf bifurcations are necessarily associated with a capital-labor ratio that is, at steady state, larger than the Golden Rule level. Consequently, paths converging asymptotically towards the steady state are shown to be dynamically inefficient, as there always exists another trajectory that starts with the same initial conditions and produces more aggregate consumption at all future dates. More surprisingly, however, our main result shows that stable orbits, generated around a dynamically inefficient steady state through a supercritical Hopf bifurcation, may in contrast be dynamically efficient. Therefore an inefficient stationary solution may coexist with an efficient invariant closed curve that surrounds it.

The interest of exploring such a topic has been dictated by the evidence that the large literature on indeterminacy in growth models has paid little attention to the Pareto-optimality properties of the allocations associated with the existence of a continuum of

\footnotetext{
${ }^{1}$ This paper has been written for the conference in honor of Jean-Michel Grandmont, held in Lisbon on october 30-31, 2005. This is our modest contribution both to celebrate JeanMichel's scientific achievements and to thank him for his impulse on our personal and professional lives. We thank participants, especially Subir Chattopadhyay, Jean-Michel Grandmont, Cars Hommes, Guy Laroque, Kazuo Nishimura, as well as a referee for helpful comments on the first version of this paper. We finally thank David de la Croix and the late Philippe Michel for discussions on the OLG model.
} 
intertemporal equilibria all converging to the stationary solution (s ee, however, Chattopadhyay and Gottardi, 1999, Montrucchio, 2004, Pietra, 2004, among many others, for studies of optimality in pure exchange economies). Since dynamic efficiency is a necessary condition for Pareto-optimality, we found important to explore how such an issue relates to indeterminate equilibria.

By studying a broad class of OLG economies, we could prove that the conclusions of Reichlin's (1986) section 3 and appendix are questionable, as the steady state, even close to the Leontief case, turns out to be always dynamically inefficient, hence not Paretooptimal. Such a result has remarkable implications as it implies that stabilization policies conducted by the governments in order to eliminate endogenous fluctuations, such as those considered by Reichlin's (1986) section 3, may lead to a dynamically inefficient steady state. Therefore, such policies do not avoid the welfare loss associated with dynamic inefficiency. On the other hand, our results suggest that simple policies that aim at restoring dynamic efficiency, through public debt or pensions for instance, may also be powerful enough to rule out indeterminacy and endogenous business cycles. We also qualify the often stated view that no general connection between indeterminacy and efficiency should be expected (e.g. Woodford, 1984, p.28).

Our analysis is fairly general so that the direct approach provided by Cass (1972) and generalized by Benveniste (1976), based on the consideration of the technological side only, can be extended to frameworks that take into consideration also the consumption side (i.e. preferences). An early paper by Cass and Yaari (1966) is a previous study along these lines, in a model with neither labor nor capital. In this paper, we build upon previous results by Pintus (1997, Props. 2.2.4 and 2.2.7) proving that the indeterminate steady state is dynamically inefficient. More recently, Lloyd-Braga et al. (2004) have considered an OLG framework and showed that local indeterminacy occurs also when the steady state is dynamically efficient. However, the latter result relies on externalities that are driven by labor only, in which case 
the definition of Golden Rule turns out to be unaltered by the presence of external effects (in contrast with the nontrivial case of capital externalities studied by Cazzavillan, 2001, and Cazzavillan and Pintus, 2004). Notice though that none of these researches establish, as we do in the present paper, that an inefficient steady state may be surrounded by efficient stable orbits.

The paper is organized as follows. Section 2 introduces the model and derives the two dimensional dynamical system that generates competitive intertemporal equilibria with perfect foresight. Section 3 establishes the link that exists between a locally indeterminate normalized steady state and Hopf bifurcations and dynamic inefficiency. In Section 4 we provide a simple example that enables to illustrate the general results stated in Section 3. Section 5 gathers the concluding remarks.

\section{THE MODEL}

We consider a competitive, non-monetary, overlapping generations model with production. The framework involves a unique perishable good, which can be either consumed or saved as investment, a large number of identical competitive firms all facing the same technology, and a constant population composed of households living two periods. Agents, who are identical within each generation, consume in both periods, supply labor and save when young. When old, their saved income is rented as physical capital to the firms.

Assuming additively separable preferences and letting $\ell_{t}$, $c_{1 t}$ and $z_{t}$ be labor, consumption and savings (i.e. capital demand), respectively, of an individual of the young generation, and $c_{2 t+1}$ be the consumption of the same individual when old, an economic agent born at time $t \geq 0$ solves the following maximization program:

$$
\operatorname{Max}\left[U_{1}\left(c_{1 t} / B\right)-U_{3}\left(\ell_{t}\right)+U_{2}\left(c_{2 t+1}\right)\right]
$$


subject to the constraints

$$
\begin{aligned}
& \quad c_{1 t}+z_{t}=\Omega_{t} \ell_{t} \\
& c_{2 t+1}=R_{t+1} z_{t} \\
& \quad c_{1 t} \geq 0,, c_{2 t+1} \geq 0, \bar{\ell} \geq \ell_{t} \geq 0, \text { for all } t \geq 0,
\end{aligned}
$$

where $\Omega_{t}>0$ and $R_{t+1}>0$ represent the real wage at time $t$ and the gross interest rate at time $t+1$, whereas $B>0$ and $\bar{\ell}$ are a scaling parameter (which will be set appropriately so as to normalize the steady state under study; see next section) and the maximum available amount of labor, respectively.

On the preferences introduced in the above maximization problem, we shall make the following assumptions.

Assumption 2.1. The functions $U_{1}(c / B), U_{2}(c)$ and $U_{3}(\ell)$ are defined and continuous on the set $\mathfrak{R}_{+}$. Moreover, they are $C^{r}$, for $r$ large enough, on the set $\mathfrak{R}_{++}$, with $\quad U_{1}^{\prime}(c / B)>0, \quad U_{2}^{\prime}(c)>0, \quad U_{3}^{\prime}(\ell)>0, \quad U_{1}^{\prime \prime}(c / B)<0, \quad U_{2}^{\prime \prime}(c)<0$, $U_{3}^{\prime \prime}(\ell)>0, \quad \operatorname{Lim}_{\ell \rightarrow \bar{\ell}} U_{3}^{\prime}(\ell)=+\infty$, where $\bar{\ell}>1$, and $\operatorname{Lim}_{\ell \rightarrow 0} U_{3}^{\prime}(\ell)=0$. In addition, $\quad 0<R_{1}(c / B) \equiv-(c / B) U_{1}^{\prime \prime}(c / B) / U_{1}^{\prime}(c / B)<1$, $0<R_{2}(c) \equiv-c U_{2}^{\prime \prime}(c) / U_{2}^{\prime}(c)<1$, and $R_{3}(\ell) \equiv \ell U_{3}^{\prime \prime}(\ell) / U_{3}^{\prime}(\ell)>0$.

The conditions $\quad 0<R_{1}(c / B)<1$ and $\quad 0<R_{2}(c)<1 \quad$ in Assumption 2.1 ensure that consumption in both periods and leisure are gross substitute, and that the saving function is increasing in the gross interest rate $R$.

When the solution of the households maximization problem is interior, the first order conditions are:

$$
U_{1}^{\prime}\left(c_{1 t} / B\right) / B=U_{3}^{\prime}\left(\ell_{t}\right) / \Omega_{t}=U_{2}^{\prime}\left(c_{2 t+1}\right) R_{t+1} .
$$

Since the function $U_{1}^{\prime}(c / B)$ is strictly decreasing, hence invertible, and $U_{3}^{\prime}(\ell)$ is onto $\mathfrak{R}_{+}$in view of Assumption 2.1, the 
first equality in Eqs. (3) can be used to obtain the expression for current consumption which, therefore, can be written as:

$$
c_{1 t}=B\left(U_{1}^{\prime}\right)^{-1}\left(B \frac{U_{3}^{\prime}\left(\ell_{t}\right)}{\Omega_{t}}\right) .
$$

From the budget constraint in Eq. (1), faced by a young agent, one gets:

$$
z_{t}=\Omega_{t} \ell_{t}-B\left(U_{1}^{\prime}\right)^{-1}\left(B \frac{U_{3}^{\prime}\left(\ell_{t}\right)}{\Omega_{t}}\right)
$$

Multiplying both members of the last equality in Eqs. (3) by $z_{t}$ yields:

$$
R_{t+1} z_{t}=u_{2}^{-1}\left(\frac{U_{3}^{\prime}\left(\ell_{t}\right)}{\Omega_{t}} z_{t}\right),
$$

where $u_{2}\left(c_{2 t+1}\right) \equiv c_{2 t+1} U_{2}^{\prime}\left(c_{2 t+1}\right)$ is strictly increasing in view of Assumption 2.1.

Output, denoted by $y$, is produced using capital $k$ and labor $\ell$. We shall assume that the technology is given by the aggregate production function $y=A F(k, \ell)$, where $A>0$ is a productivity scaling factor. The production function satisfies the following properties.

Assumption 2.2. $F(k, \ell)$ is defined, continuous, strictly concave on the set $\mathfrak{R}_{+}^{2}$, and homogeneous of degree one, i.e. $F(k, \ell) \equiv \ell f(a)$, with $a \equiv k / \ell$. Moreover, $f^{\prime}(a)>0$ and $f^{\prime \prime}(a)<0$, for all $a>0$. It follows that the functions $\rho(a) \equiv f^{\prime}(a)>0$ and $\omega(a) \equiv f(a)-a f^{\prime}(a)>0 \quad$ are, $\quad$ respectively, decreasing and increasing. 
Each firm, which behaves competitively, seeks to maximize profits taking factor prices as given. Letting $0 \leq \delta \leq 1$ and $q=R+\delta-1>0$ be the (constant) per-period depreciation rate and the rental on capital stock respectively, equilibrium factor prices are given by

$$
\Omega=A \omega(a) \equiv \Omega(a), \quad R=A \rho(a)+1-\delta \equiv R(a) .
$$

Eqs. (5), (6) and (7) can be combined to derive a twodimensional dynamical system described below in Eqs. (8) and (9) which generates the intertemporal equilibria with perfect foresight of the economy under study:

$$
\begin{aligned}
k_{t+1}=\Omega\left(a_{t}\right) \frac{k_{t}}{a_{t}}-B\left(U_{1}^{\prime}\right)^{-1}\left(\frac{B U_{3}^{\prime}\left(k_{t} / a_{t}\right)}{\Omega\left(a_{t}\right)}\right), \\
R\left(a_{t+1}\right) k_{t+1}=u_{2}^{-1}\left(\frac{U_{3}^{\prime}\left(k_{t} / a_{t}\right)}{\Omega\left(a_{t}\right)} k_{t+1}\right) .
\end{aligned}
$$

\section{LOCAL INDETERMINACY, HOPF BIFURCATIONS AND DYNAMIC INEFFICIENCY}

The dynamical system (8)-(9) has an interior steady-state (not necessarily unique) if the following holds.

PROPOSITION 3.1. Under Assumptions 2.1 and $2.2,\left(a^{*}, k^{*}\right)>0$ is a steady-state of the dynamical system (8)-(9) if and only if:

$$
\begin{aligned}
& a^{*} u_{2}\left(k^{*}\left(\frac{a^{*} \rho\left(a^{*}\right)}{\omega\left(a^{*}\right)}+1-\delta\right)\right)<k^{*} U_{3}^{\prime}\left(k^{*} / a^{*}\right) \text { and } \\
& \operatorname{Lim}_{c \rightarrow 0} c U_{1}^{\prime}(c)<\frac{k^{*}\left(A^{*} \omega\left(a^{*}\right) / a^{*}-1\right)}{A^{*} \omega\left(a^{*}\right)} U_{3}^{\prime}\left(k^{*} / a^{*}\right),
\end{aligned}
$$


where $A^{*}>a^{*} / \omega\left(a^{*}\right) \quad$ is the unique solution of $k^{*}\left(A \rho\left(a^{*}\right)+1-\delta\right)=u_{2}^{-1}\left(k^{*} \frac{U_{3}^{\prime}\left(k^{*} / a^{*}\right)}{A \omega\left(a^{*}\right)}\right)$.

Proof. See Appendix A.

To characterize the local dynamics generated by the dynamical system (8) and (9), one may focus on the determinant and the trace of the linear map associated with the Jacobian matrix evaluated at steady-state $\left(a^{*}, k^{*}\right)>0$ whose existence, according to Proposition 3.1, will be assumed throughout the section. Let $\varepsilon_{\Omega}$ and $\varepsilon_{R}$ be the elasticities of the functions $\Omega(a)$ and $R(a)$ evaluated at the steady-state $\left(a^{*}, k^{*}\right)>0$. In addition, let $\theta \equiv \Omega\left(a^{*}\right) / a^{*}=A^{*} \omega\left(a^{*}\right) / a^{*}>1 \quad$ (c.d. Proposition $3.1 \quad$ above) $R_{1} \equiv R_{1}\left(c_{1}^{*} / B^{*}\right), \quad R_{2} \equiv R_{2}\left(c_{2}^{*}\right), \quad$ and $\quad R_{3} \equiv R_{3}\left(\ell^{*}\right) . \quad$ Then, the expressions of the trace $\mathrm{T}$ and the determinant $\mathrm{D}$ are given by:

$$
D=\theta \frac{\varepsilon_{\Omega}}{\left|\varepsilon_{R}\right|}\left(\frac{1+R_{3}}{1-R_{2}}\right)
$$

and

$$
T=\theta+\frac{\theta-1}{R_{1}} R_{3}+\frac{1}{\left(1-R_{2}\right)\left|\varepsilon_{R}\right|}\left[R_{3}+\varepsilon_{\Omega}+R_{2}\left(\frac{\theta-1}{R_{1}}\left(R_{3}+\varepsilon_{\Omega}\right)-\theta\left(\varepsilon_{\Omega}-1\right)\right)\right]
$$

where the elasticities of $\Omega(a)$ and $R(a)$ can be rewritten as

$$
\varepsilon_{\Omega}=\frac{s(a)}{\sigma(a)},\left|\varepsilon_{R}\right|=\mu(a)\left(\frac{1-s(a)}{\sigma(a)}\right),{ }^{2}
$$

\footnotetext{
${ }^{2}$ One can easily check that $\Omega^{\prime}(a)=-a q^{\prime}(a)$ and, therefore, that $a \rho(a) / f(a)=\sigma(a) \varepsilon_{\Omega}(a)$ and $\omega(a) / f(a)=\sigma(a)\left|\varepsilon_{q}(a)\right|$, where the elasticity of input substitution is
} 
with $0<\mu(a) \equiv q(a) / R(a)=s(a) \theta(a) /(s(a) \theta(a)+(1-s(a))(1-\delta)) \leq 1$.

The local dynamic analysis of the model has already been done in Cazzavillan and Pintus (2004). Therefore we shall refer the reader to that paper for details. To help interpretation, it is useful to relate the parameter $\theta \geq 1$ to the consumption-to-wage ratio: Eq (1) shows that $c_{1} /(\Omega \ell)=(\theta-1) / \theta$ (alternatively, $k /(\Omega \ell)=1 / \theta$ is the ratio of savings over wage income). In particular, one can recover the results by Reichlin (1986), when $\delta=1$, and by Grandmont (1993) when restricting to the case $\theta=1$ (no consumption in the first period of life) and $\sigma=0$ (Leontief technology). It is perhaps worthwhile to remind, from Cazzavillan and Pintus (2004), that the steady state is locally indeterminate when the propensity to consume out of the wage income is small, that is, when $\theta$ is close enough to one (and also when the share of capital in total income is sufficiently low, the elasticity of labor supply with respect to real wage is large enough, the first-period utility is close to logarithmic, the second-period utility is close to linear, and the elasticity of input substitution is small). All these features are obviously related to the conditions leading to local indeterminacy in the model studied by Reichlin (1986) and Grandmont (1993), that can be considered as a limit case of our setting involving consumption in both periods.

For the purpose of the present investigation it is important to stess that there are dynamic regimes in which the stationary solution $\left(a^{*}, k^{*}\right)>0$ is a sink and then undergoes a Hopf bifurcation as the selected bifurcation parameter (say $\phi$ ) is increased while $\sigma(a)=\left(\varepsilon_{\Omega}(a)-\varepsilon_{q}(a)\right)^{-1} \geq 0$ with $\varepsilon_{\Omega}(a)$ and $\varepsilon_{q}(a)$ representing the elasticities of the real wage $\Omega(a)$ and of the rental on capital $q(a)=R(a)-1+\delta$. In addition, one also gets $0<\mu(a) \equiv q(a) / R(a)=s(a) \theta(a) /(s(a) \theta(a)+(1-s(a))(1-\delta)) \leq 1 . \quad$ Rearranging $\quad$ these expressions yields the elasticities in (12). 
keeping all the others fixed (c.d. Cazzavillan and Pintus pp. 46668). On the basis of such a knowledge, we can relate the existence of both a locally indeterminate steady state and periodic or quasiperiodic orbits to dynamic inefficiency. The next proposition establishes a first general result.

Following Phelps (1965), we first define the Golden Rule level of the capital-labor ratio.

Definition 3.1. Under the assumptions of Proposition 3.1, there exists a unique capital-labor ratio such that $A^{*} f^{\prime}\left(a_{G R}^{*}\right)=\delta$, provided that $\operatorname{Lim}_{a \rightarrow+\infty} A^{*} f^{\prime}(a)<\delta<\operatorname{Lim}_{a \rightarrow 0} A^{*} f^{\prime}(a) . \quad$ The level $a_{G R}^{*}$ is the Golden Rule capital-labor ratio.

The level $a_{G R}^{*}$ characterizes productive efficiency. By the assumption of market clearing, one has that stationary aggregate consumption equals net production, that is, $c_{1}^{*}+c_{2}^{*}=A^{*} \ell^{*} f\left(k^{*} / \ell^{*}\right)-\delta k^{*}$. Production efficiency is then characterized by $A^{*} f^{\prime}\left(a_{G R}^{*}\right)=\delta$, which defines the Golden Rule steady state capital-labor ratio $a_{G R}^{*}$, under the assumptions of Definition 3.1.

PROPOSITION 3.2. Under the assumptions of Definition 3.1, local indeterminacy and Hopf bifurcation of the steady state $\left(a^{*}, k^{*}\right)>0$ imply $a^{*}>a_{G R}^{*}$, or equivalently $A^{*} f^{\prime}\left(a^{*}\right)<\delta$, that is, the stationary capital-labor ratio is larger than the Golden Rule level.

Proof. See Appendix B.

The statement in the above proposition is in contrast with Reichlin's (1986) appendix and arguably casts some doubt on the desirability of stabilization policies pushing the economy towards the steady state, as defined in his section 3 . However, the result is 
in agreement with the intuition that both local indeterminacy and Hopf bifurcations rely on high savings rates (that is, on large values of $1 / \theta)$. When, on the contrary, the savings rate is low, agents arbitrage away expectation-driven business cycles. For sake of brevity, we mention here, without formal proof, the fact that a dynamically efficient steady state is always a saddle point in the class of models under study.

We next define, after Phelps (1965) and Diamond (1965), the Golden Age.

Definition 3.2. Under the assumptions of Definition 3.1, there exists a unique stationary allocation, defined as the Golden Age, with $a^{*}=a_{G R}^{*}, c_{1}^{*}=c_{1 G R}^{*}$, $c_{2}^{*}=c_{2 G R}^{*}, \ell^{*}=\ell_{G R}^{*}$, and

$$
U_{1}^{\prime}\left(c_{1 G R}^{*} / B^{*}\right) / B^{*}=U_{3}^{\prime}\left(\ell_{G R}^{*}\right) / \Omega\left(a_{G R}^{*}\right)=U_{2}^{\prime}\left(c_{2 G R}^{*}\right) .
$$

It is straightforward to show that the Golden Age characterizes steady state Pareto-optimality, which also implies that the Golden Rule level is achieved.

Our task now is to examine the dynamic (in)efficiency of orbits around the normalized steady state by looking at the paths of capital and labor. Here adapting the direct approach advocated by Cass (1972) proves to be much useful. Denoting $\left\{k_{t}\right\}=\left\{k_{0}, k_{1}, \cdots\right\}$ and $\left\{\ell_{t}\right\}=\left\{\ell_{0}, \ell_{1}, \cdots\right\}$, with $a \equiv k / \ell$, a particular path solving Eqs. (8)(9), one has:

Definition 3.2. A path of the capital stock $\left\{k_{t}^{0}\right\}$ is said to be dynamically inefficient (resp. efficient) if and only if there is, given $\left\{\ell_{t}\right\}$, some (resp. no) other path $\left\{k_{t}^{1}\right\}$ which provides more consumption for some periods and never less consumption, or equivalently $C\left(k_{t}^{1}, k_{t+1}^{1}, \ell_{t}\right) \geq C\left(k_{t}^{0}, k_{t+1}^{0}, \ell_{t}\right)$, with strict inequality for some $\mathrm{t}$, where 
$C\left(k_{t}, k_{t+1}, \ell_{t}\right) \equiv A^{*} F\left(k_{t}, \ell_{t}\right)-k_{t+1}+(1-\delta) k_{t}$ defines

total

consumption in date $t$.

The following lemma, adapted from Cass (1972, Props 1 and 4), provides a convenient necessary and sufficient condition for dynamic inefficiency.

LEMMA 3.1. A path of the capital stock $\left\{k_{t}\right\}$ is inefficient if and only if, given $\left\{\ell_{t}\right\}$, there exists a sequence of capital stock decrements $\left\{\varepsilon_{t}\right\}=\left\{\varepsilon_{0}, \varepsilon_{1}, \cdots\right\}$ such that

$$
\varepsilon_{t+1} \geq \tilde{F}_{k}\left(k_{t}-\varepsilon_{t}, \ell_{t}\right) \varepsilon_{t} \quad \text { and } \quad \varepsilon_{t}>0
$$

where $\tilde{F}(k, \ell) \equiv A^{*} F(k, \ell)+(1-\delta) k$ and $\tilde{F}_{k}(\cdot) \equiv \partial \tilde{F}(\cdot) / \partial k$.

To save space, the proof is omitted, as it follows from adapting the proofs of Propositions 1 and 4 in Cass (1972).

The next Proposition follows as a mere implication of Proposition 3.2 .

PROPOSITION 3.3. Let $\phi$ be the selected bifurcation parameter. Then, under the assumptions of Proposition 3.1, the following holds.

(i) If the steady state $\left(a^{*}, k^{*}\right)>0$ is a sink (hence locally indeterminate), there exists a non-empty neighborhood $W$ of $\left(a^{*}, k^{*}\right)>0$ such that, for all initial conditions $\left(a_{0}, k_{0}\right) \in W$, intertemporal equilibria are dynamically inefficient.

(ii) If the steady state $\left(a^{*}, k^{*}\right)>0$ undergoes a subcritical Hopf bifurcation at the parameter value $\phi=\phi_{H}$, there 
exist both a non-empty interval $I \equiv\left(\phi_{H}-\varepsilon, \phi_{H}\right)$, for some $\varepsilon>0$, and a non-empty neighborhood $W$ of $\left(a^{*}, k^{*}\right)>0$ contained in the interior of the unstable Hopf curve such that, for all $\phi \in I$ and all initial conditions $\left(a_{0}, k_{0}\right) \in W$, intertemporal equilibria are dynamically inefficient.

Proof. See Appendix C.

Case (ii) in Proposition 3.3 covers the case where the neighborhood $W$ of the steady state shrinks when the bifurcation parameter $\phi$ tends, from below, to the subcritical Hopf bifurcation value $\phi_{H}$.

To show that allocations that are associated with cases (i)(ii) are not Pareto-optimal is straightforward, as dynamic efficiency is easily proved to be a necessary condition of Pareto-optimality of consumption levels along the life-cycle that turns out to be unsatisfied here. In particular, Proposition 3.3 involves a neighborhood that does not intersect the "Golden rule frontier" $a=a_{G R}^{*}$ (see Fig. 2 below). However, we now state some conditions under which endogenous cycles are dynamically efficient and coexist with a dynamically inefficient steady state $\left(a^{*}, k^{*}\right)>0$. In addition, in the next paragraph a specific example will be provided to show how such a result can be generated.

PROPOSITION 3.4. Under the assumptions of Proposition 3.1, suppose that the steady state $\left(a^{*}, k^{*}\right)>0$ undergoes a supercritical Hopf bifurcation at $\phi=\phi_{H}$. Then one gets the following:

(i) If the Hopf curve contains periodic orbits with period $\tau$, intertemporal equilibria are dynamically efficient if and only if $\prod_{s=0}^{\tau-1} R_{s} \geq 1$ 
(ii) If the Hopf curve contains quasiperiodic orbits, intertemporal equilibria are dynamically efficient if and only if $\operatorname{Lim}_{t \rightarrow+\infty} \sum_{s=0}^{t} \prod_{r=0}^{s-1} R_{r}=+\infty$,

where $R_{t} \equiv \tilde{F}_{k}\left(k_{t}, \ell_{t}\right)=A^{*} F_{k}\left(k_{t}, \ell_{t}\right)+1-\delta$.

Proof. See Appendix D.

One implication of (i)-(ii) in Proposition 3.4 is that dynamic efficiency obtains, when $\phi \in I \equiv\left(\phi_{H}, \phi_{H}+\varepsilon\right)$ with some $\varepsilon>0$, only if the stable Hopf curve surrounding the unstable steady state has a non-empty intersection with the Golden Rule frontier $a=a_{G R}^{*}$ (see Fig. 3 below), as we now see in an example.

\section{AN EXAMPLE}

For illustrative purpose we now present a simple example which will allow to appreciate the qualitative behavior of the local dynamics as well as the intuition that rely upon the link between indeterminacy and dynamic inefficiency. To the non-specialized reader it is worthwile to recall how a simple way to analyze the local stability of the steady-state $\left(a^{*}, k^{*}\right)>0$ is to study the variation of the trace $T$ and the determinant $D$ in the $(T, D)$ plane when some parameters of interest are made to vary continuously (see Grandmont and Laroque, 1993, Azariadis, 1993, Grandmont, Pintus and de Vilder, 1998). There is a local eigenvalue equal to + 1 when $1-T+D=0$, which is represented by the line (AC) in Fig. 1. On the other hand, one eigenvalue is equal to - 1 when $1+T+D=0$, i.e. when $(T, D)$ lies on the line (AB). Finally, the two eigenvalues are complex conjugate of modulus 1 , when $(T, D)$ belongs to the segment [BC] of equation $D=1,|T| \leq 2$. Since both characteristic roots are equal to zero when $T=D=0$, then, by continuity, they have modulus less than one if and only if $(T, D)$ 
belongs to the interior of the triangle $\mathrm{ABC}$ (see Fig. 1) defined by $|T|<|1+D|,|D|<1$. In this case the steady-state is asymptotically stable in the forward perfect foresight dynamics, i.e. locally indeterminate given that the unique predeterminate variable is $k$. When $|T|>|1+D|$, instead, the stationary state is a saddle-point, hence locally determinate. Finally, in the complementary region $|T|<|1+D|,|D|>1$, the steady-state is a source, hence locally determinate.

\section{Insert Fig. 1 here}

Furthermore, the diagram reported in Fig. 1 is also useful to study local bifurcations. When the point $(T, D)$ crosses the interior of the segment $[\mathrm{BC}]$, one generically expects a Hopf bifurcation, i.e. a change of stability in which the eigenvalues are complex conjugate and cross the unit circle in the complex plane. If, instead, the point crosses the line (AB), one eigenvalue goes through - 1. In that case, a flip bifurcation is expected, generically, to occur. Finally, when the point crosses the line (AC), one eigenvalue goes through +1 . In such a case, one generally expects an exchange of stability between $\left(a^{*}, k^{*}\right)>0$ and another (respectively two) steadystate(s) through a transcritical bifurcation (respectively a pitchfork bifurcation).

One can then easily look at local stability and bifurcations simply by locating the half-line $\Delta$ in the $(T, D)$ plane when a parameter varies while all the others are kept fixed.

In the present context we simplify matters by assuming that $R_{2}=\sigma=0$ and by holding fixed all parameters with the exception of $R_{3}$ which is free to increase from 0 to $+\infty$. In that case, according to Eqs. (10)-(12), the expressions of the determinant and the trace are: 


$$
\begin{gathered}
D\left(R_{3}\right)=\theta \frac{s}{\mu(1-s)}\left(1+R_{3}\right)>0 \quad \text { and } \\
T\left(R_{3}\right)=\theta+\frac{\theta-1}{R_{1}} R_{3}+\frac{s}{\mu(1-s)}>1,
\end{gathered}
$$

with $\mu=(s \theta) /(s \theta+(1-s)(1-\delta))$. The $\Delta$ line is then generated when $R_{3}$ increases from 0 to $+\infty$. In particular, the origin of $\Delta$ is located on the line (AC) at the point of coordinates $(T(0), D(0))=(\theta+s /[\mu(1-s)],(s \theta) /[\mu(1-s)])$, whereas its slope is equal to $\left(s \theta R_{1}\right) /(\mu(1-s)(\theta-1))$ and decreases from $+\infty$ to $s R_{1} /(1-s)$ when $\theta$ increases from one to $+\infty$. From direct inspection one can see that local indeterminacy and Hopf bifurcation obtain, by construction, if $D(0)<1$, that is if $s<\delta(1-s)$ when $\theta=1$. One also sees that the slope of the half-line $\Delta$ is decreasing with $\theta$ from $+\infty$, when $\theta=1$, to $\left(s R_{1}\right) /(1-s)$ which is less than 1 provided that $D(0)<1$. As for the origin along the (AC) line, it is easy to verify that it moves with $\theta$ along a line $\Delta_{1}$ the slope of which is $s /(\mu(1-s))$. The latter is less than 1 whenever $\mathrm{D}(0)<1$, i.e. $s<\delta(1-s)$.

As a result, provided that the inequality $s<\delta(1-s)$ holds, one obtains the configuration shown in Fig. 1. Notice that, by continuity, as $\theta$ is moved from 1 to $+\infty$, there exists a $\theta^{c}$ such that $\Delta$ goes through the point $\mathrm{C}$. It follows that one obtains local indeterminacy and Hopf bifurcations only in the interval $\left[1, \theta^{c}\right)$. The corresponding bifurcation point within that interval is found by solving $D\left(R_{3}\right)=1$ and yields the bifurcation value $R_{3 H}=(\mu(1-s)) /(\theta s)-1$. Consequently, being such a regime nonempty, we can now focus on the results stated in Proposition 3.4.

Suppose that: 


$$
U_{1}\left(c_{1}\right)=\frac{\left(c_{1} / B\right)^{1-\alpha_{1}}}{1-\alpha_{1}}, \quad U_{2}\left(c_{2}\right)=c_{2}, \quad U_{3}(\ell)=\frac{\ell^{1+\alpha_{3}}}{1+\alpha_{3}}
$$

where $0 \leq \alpha_{1} \leq 1, \alpha_{3} \geq 0$, and $B>0$. In addition, let the intensive production function be

$$
f(a)=A\left(s a^{-\rho}+1-s\right)^{-1 / \rho} \text {, with } \rho \geq-1 .
$$

In view of Proposition 3.1 , one can set the steady state $\left(a^{*}, k^{*}\right)$ at $(1,1)$ provided that

$$
A^{*}=[s(1-s)]^{-1 / 2}
$$

and

$$
B^{*}=\left[(1-s) A^{*}\left((1-s) A^{*}-1\right)^{-\alpha_{1}}\right]^{\frac{1}{1-\alpha_{1}}} .
$$

One can then get the expression of the golden rule through simply algebra and obtain:

$$
a_{G R}^{*}=\left[\left(\left(s A^{*} \delta^{-1}\right)^{\rho /(1+\rho)}-s\right) /(1-s)\right]^{1 / \rho} .
$$

Notice that $a_{G R}^{*}$ tends to one when $\rho \rightarrow+\infty$ if $A^{*}>\delta$ (a condition that holds when $s=1 / 3)$. It follows that $a^{*}=a_{G R}^{*}$ holds in the limiting Leontief case. For the specific example studied in this section we have checked, by using the DUNRO program elaborated by Sands and de Vilder, that the (locally indeterminate) normalized steady state undergoes a supercritical Hopf bifurcation.

It is then possible to give the reader a complete qualitative overview of what happens in a neighboorhood of the stationary solution and illustrate case (i) as stated in Proposition 3.4.

\section{Insert Fig. 2 and Fig. 3 here}


Figures 2 and 3 represent, in the $(a, k)$ plane, the normalized steady state $\left(a_{G R}^{*}, k^{*}\right)$, a period-3 cycle, and a dashed area in which dynamic inefficiency prevails, i.e. the region where $A^{*} f^{\prime}(a)<\delta$ or, equivalently, $a>a_{G R}^{*}$. In both pictures the steady state is unstable, hence locally determinate, while the period-3 cycle is stable for some $R_{3}>R_{3 H}$. Let $R^{i} \equiv A^{*} f^{\prime}\left(a^{i}\right)+1-\delta$, for $i=1,2,3$, define the real gross interest rate evaluated at point $i$. Hence one gets, as reported in Fig. 2, that a dynamically inefficient period-3 cycle obtains when $R^{1} R^{2} R^{3}<1$, that is when the points 2 and 3 located in the dynamic inefficiency region "dominate" the point 1 . On the contrary, Fig. 3 depicts a dynamically efficient period-3 cycle provided that $R^{1} R^{2} R^{3} \geq 1$. This is again the case where the points 2 and 3 "dominate" the point 1 .

\section{CONCLUDING REMARKS}

This paper has explored how the occurrence of local indeterminacy and endogenous business cycles relates to dynamic inefficiency, in a standard two-period OLG model of capital accumulation with labor-leisure choice into the first-period of agents' life and consumption in both periods. We have shown that local indeterminacy and subcritical Hopf bifurcations are necessarily associated with paths converging asymptotically towards the steady state that are dynamically inefficient. Most interesting is our main result showing how stable orbits, generated around a dynamically inefficient steady state through a supercritical Hopf bifurcation, may, in contrast, be dynamically efficient. Therefore an inefficient stationary solution may coexist with an efficient invariant closed curve that surrounds it. One also expects that sunspots moving the economy away from an inefficient steady state for a sufficiently long time may not be inefficient.

Such a result has nontrivial implications as it implies that stabilization policies conducted by the governments in order to 
eliminate endogenous fluctuations, such as those considered by Reichlin (1986), may lead to a dynamically inefficient steady state. On the other hand, our results suggest that simple policies restoring dynamic efficiency, through public debt or pensions for instance, may be powerful enough to rule out indeterminacy and endogenous business cycles. A systematic investigation of such policies should be the subject of future research.

\section{APPENDIX}

\section{A. Proof of Proposition 3.1}

The solution $(a, k)$, with $a>0$ and $k>0$, is a steady-state of Eqs. (8)-(9) if and only if:

$$
\begin{aligned}
& k=A k \omega(a) / a-B\left(U_{1}^{\prime}\right)^{-1}\left(B \frac{U_{3}^{\prime}(k / a)}{A \omega(a)}\right), \\
& k(A \rho(a)+1-\delta)=u_{2}^{-1}\left(k \frac{U_{3}^{\prime}(k / a)}{A \omega(a)}\right) .
\end{aligned}
$$

Eq. (A.2) can be conveniently rewritten as

$A \omega(a) u_{2}(A k \rho(a)+(1-\delta) k)=k U_{3}^{\prime}(k / a)$.

Under Assumption 2.2, both $\omega(a)$ and $\rho(a)$ are finite. On the other hand, under Assumption 2.1, the left-hand side of Eq. (A.3) is increasing in $A$, as $u_{2} \equiv c U_{2}^{\prime}(c)$ is increasing when $R_{2}(c)<1$, for all $c>0$, whereas the right-hand side is constant and finite (as long as $\bar{\ell}>k / a)$. Whenever it exists, then, there is a unique positive $A^{*}$ which satisfies Eq. (A.3). Moreover, one easily shows that the first-period consumption at the steady-state $(a, k)$ is positive if and 
only if the so-computed $A^{*}$ is such that $A^{*}>a / \omega(a)$. Existence, therefore, requires the following boundary condition:

$$
a u_{2}\left(k\left(\frac{a \rho(a)}{\omega(a)}+1-\delta\right)\right)<k U_{3}^{\prime}(k / a)
$$

Next, we want to solve Eq. (A.1) for $B$, given $A=A^{*}$. Such an equation can be rearranged to get:

$\frac{k\left(A^{*} \omega(a) / a-1\right)}{B} U_{1}^{\prime}\left(\frac{k\left(A^{*} \omega(a) / a-1\right)}{B}\right)=\frac{k\left(A^{*} \omega(a) / a-1\right)}{A^{*} \omega(a)} U_{3}^{\prime}(k / a)$. (A.5)

From direct inspection, one sees that, as long as $R_{1}(c)<1$, for all $c>0$, as postulated in Assumption 2.1, the left-hand side of Eq. (A.5) is decreasing in $B$ and tends to infinity when $B$ goes to zero. Therefore, by imposing the boundary condition

$$
\operatorname{Lim}_{c \rightarrow 0} c U_{1}^{\prime}(c)<\frac{k\left(A^{*} \omega(a) / a-1\right)}{A^{*} \omega(a)} U_{3}^{\prime}(k / a)
$$

it is possible to ensure the existence of a unique $B^{*}>0$ which solves Eq. (A.5), given $A=A^{*}$. As a result, there are unique constants $A^{*}>0$ and $B^{*}>0$ such that $(a, k)$ is a stationary solution of the system (8)-(9) if and only if the two inequalities (A.4) and (A.6) are satisfied. Q.E.D.

\section{B. Proof of Proposition 3.2}

Local indeterminacy and Hopf bifurcation of the normalized steady state necessarily require $D \leq 1$. From Eq. (10), $D=\theta \frac{\varepsilon_{\Omega}}{\left|\varepsilon_{R}\right|}\left(\frac{1+R_{3}}{1-R_{2}}\right)$ 
so that $D \leq 1$ implies $\theta<\left|\varepsilon_{R}\right| / \varepsilon_{\Omega}$. The latter inequality may be rewritten as $\theta<\bar{\theta} \equiv \delta\left(1-s\left(a^{*}\right)\right) / s\left(a^{*}\right)$. Recalling that by definition $\theta=A^{*} \ell^{*} \omega\left(a^{*}\right) / k^{*}, \quad$ one has that $\theta=A^{*} \ell \omega\left(a^{*}\right) / k^{*}=A^{*} \rho\left(a^{*}\right)\left(\ell^{*} \omega\left(a^{*}\right) /\left(k^{*} \rho\left(a^{*}\right)\right) \quad\right.$ or $\theta=A^{*} f^{\prime}\left(a^{*}\right)\left(1-s\left(a^{*}\right)\right) / s\left(a^{*}\right) . \quad$ Therefore, one gets that $\theta<\bar{\theta}$ is equivalent to $A^{*} f^{\prime}\left(a^{*}\right)<\delta \equiv A^{*} f^{\prime}\left(a_{G R}^{*}\right)$ or, more simply, $a^{*}>a_{G R}^{*}$. When either local indeterminacy or Hopf bifurcation of the steady state occurs, the stationary value of the capital-labor ratio is necessarily larger than the Golden Rule level. Q.E.D.

\section{Proof of Proposition 3.3}

We know from Proposition 3.2 that both local indeterminacy and Hopf bifurcation of the steady state $\left(a^{*}, k^{*}\right)>0$ are associated with over-accumulation with respect to the golden rule, at the steady state. To show the dynamic inefficiency of paths converging to the steady state, we now simply prove that there exists, in the positive orthant of the plane, a neighborhood $W$ of $\left(a^{*}, k^{*}\right)>0$ which has an empty intersection with the Golden Rule frontier $a=a_{G R}^{*}$. We show that, for all initial conditions $\left(a_{0}, k_{0}\right) \in W$, aggregate consumption can be increased forever, starting at any date $t_{0}$, by lowering the capital stock at $t_{0}$.

Suppose first that such a neighborhood $W$ exists. Then for all $\left(a_{0}, k_{0}\right) \in W$ and all $t \geq t_{0}$, one then has $A^{*} F_{k}(k-\varepsilon, \ell)<\delta$ for some $\varepsilon>0$, that is, over-accumulation prevails. Recalling that aggregate consumption equals net production minus investment, we may define the former quantity as: 
$C\left(k_{t}, k_{t+1}, \ell_{t}\right) \equiv A^{*} F\left(k_{t}, \ell_{t}\right)-k_{t+1}+(1-\delta) k_{t}$.

Suppose that we reduce, at date $t_{0}$, the capital stock $k_{t+1}$ by $\varepsilon$ so that aggregate consumption at date $t_{0}$ is increased by $\varepsilon$. Then, for all $t>t_{0}$, aggregate consumption is given by :

$C\left(k_{t}-\varepsilon, k_{t+1}-\varepsilon, \ell_{t}\right) \equiv A^{*} F\left(k_{t}-\varepsilon, \ell_{t}\right)-\left(k_{t+1}-\varepsilon\right)+(1-\delta)\left(k_{t}-\varepsilon\right)$

By concavity of production (Assumption 2.2), one has: $F(k-\varepsilon, \ell) \geq F(k, \ell)-\varepsilon F_{k}(k-\varepsilon, \ell)$.

Therefore, it follows that:

$$
\begin{gathered}
C\left(k_{t}-\varepsilon, k_{t+1}-\varepsilon, \ell_{t}\right) \equiv A^{*} F\left(k_{t}-\varepsilon, \ell_{t}\right)-\left(k_{t+1}-\varepsilon\right)+(1-\delta)\left(k_{t}-\varepsilon\right) \\
\geq A^{*} F\left(k_{t}, \ell_{t}\right)-k_{t+1}+(1-\delta) k_{t}+\varepsilon\left(\delta-A^{*} F_{k}\left(k_{t}-\varepsilon, \ell_{t}\right)\right) \\
>A^{*} F\left(k_{t}, \ell_{t}\right)-k_{t+1}+(1-\delta) k_{t},
\end{gathered}
$$

where the latter inequality follows from the fact that $A^{*} F_{k}(k-\varepsilon, \ell)<\delta$ in the neighborhood $W$ of the steady state. Consequently, aggregate consumption will be higher for all $t \geq t_{0}$ : the new path produces more consumption for the young and/or for the old agents all the time. Notice that an alternative proof would rely on Lemma 3.1: we have just shown that there exists a sequence of constant capital stock decrements $\left\{\varepsilon_{t}\right\}=\{\varepsilon, \varepsilon, \cdots\}$ solving $\varepsilon_{t+1} \geq \tilde{F}_{k}\left(k_{t}-\varepsilon_{t}, \ell_{t}\right) \varepsilon_{t}$, as $\tilde{F}_{k}(k-\varepsilon, \ell)<1$ or $A^{*} F_{k}(k-\varepsilon, \ell)<\delta$ around the dynamically inefficient steady state.

Our task is now to prove that such a neighborhood of the steady state $W$ exists in both cases covered by the Proposition. 
(i) The steady state is a sink: in consequence, one can take $W$ as an open ball centered at $\left(a^{*}, k^{*}\right)$ with a radius appropriately chosen, that is, such that $W$ has no intersection with the Golden Rule frontier $a=a_{G R}^{*}$.

(ii) The steady state undergoes a subcritical Hopf bifurcation: we know that this may occur only at $\phi=\phi_{H}$. Therefore, for all $\phi \in I \equiv\left(\phi_{H}-\varepsilon, \phi_{H}\right)$ with some $\varepsilon>0$, one can take $W$ as an open ball centered at $\left(a^{*}, k^{*}\right)$ and contained in the interior of the unstable Hopf curve surrounding the stable steady state, with a radius such that $W$ has no intersection with the Golden Rule frontier $a=a_{G R}^{*}$.

Q.E.D.

\section{Proof of Proposition 3.4}

The steady state undergoes a supercritical Hopf bifurcation: we know that this may occur only at $\phi=\phi_{H}$. A direct proof of (i) and (ii) follows by applying some results that are due to Cass (1972).

(i) When the Hopf orbit is periodic with period $\tau$, we know from Cass (1972, p. 214) that the partial criterion contained in his Theorem 2 turns out to be complete. More precisely, periodic orbits are shown to be inefficient if and only if $\prod_{s=0}^{\tau-1} R_{s}<1$.

(ii) When the Hopf orbit is quasiperiodic, we may apply Theorem 3 in Cass (1972, p. 209) to show that orbits are inefficient if and only if $\operatorname{Lim}_{t \rightarrow+\infty} \sum_{s=0}^{t} \prod_{r=0}^{s-1} R_{r}<+\infty$. Q.E.D. 


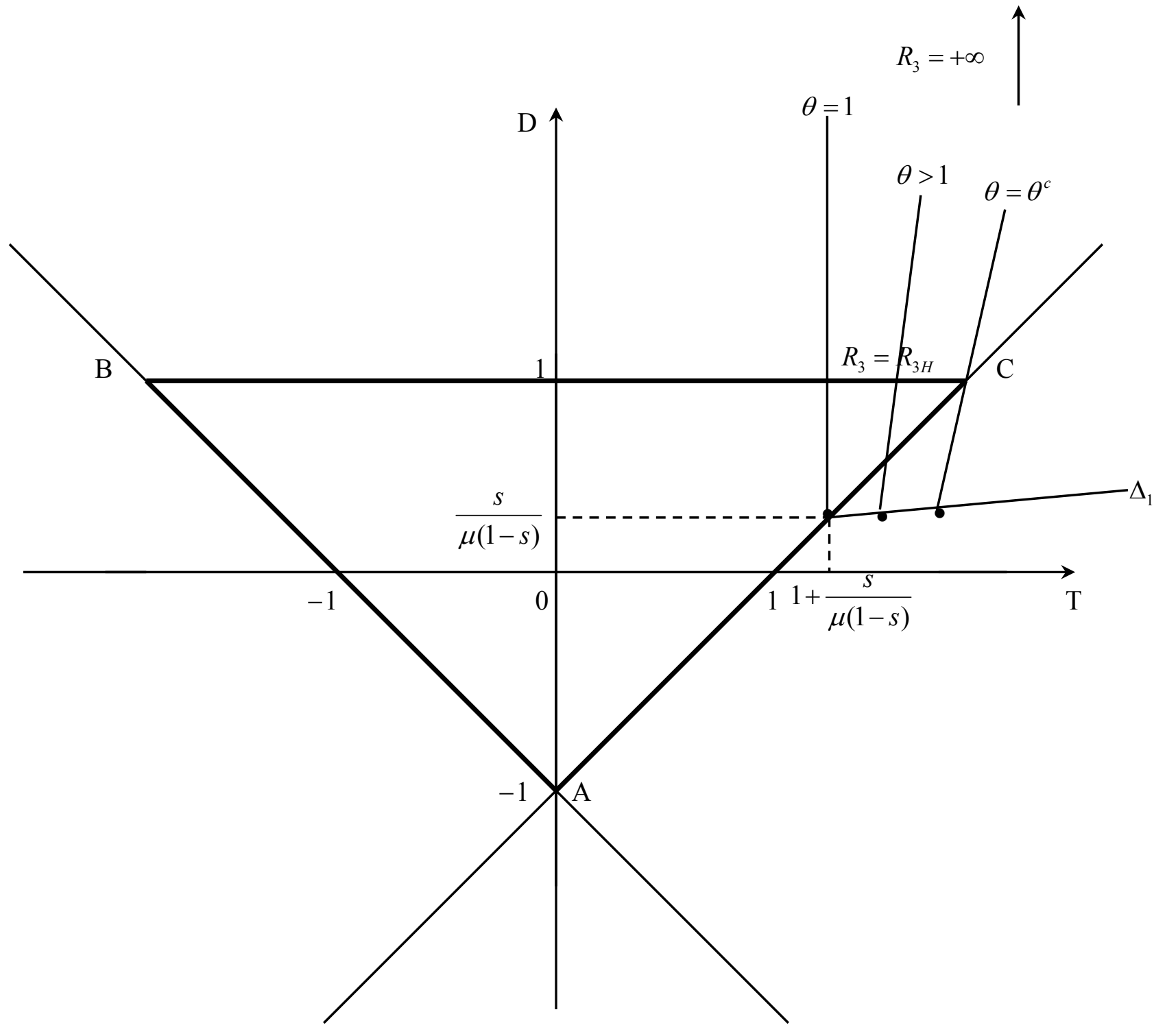

Fig.1 An example with Leontief technology. 


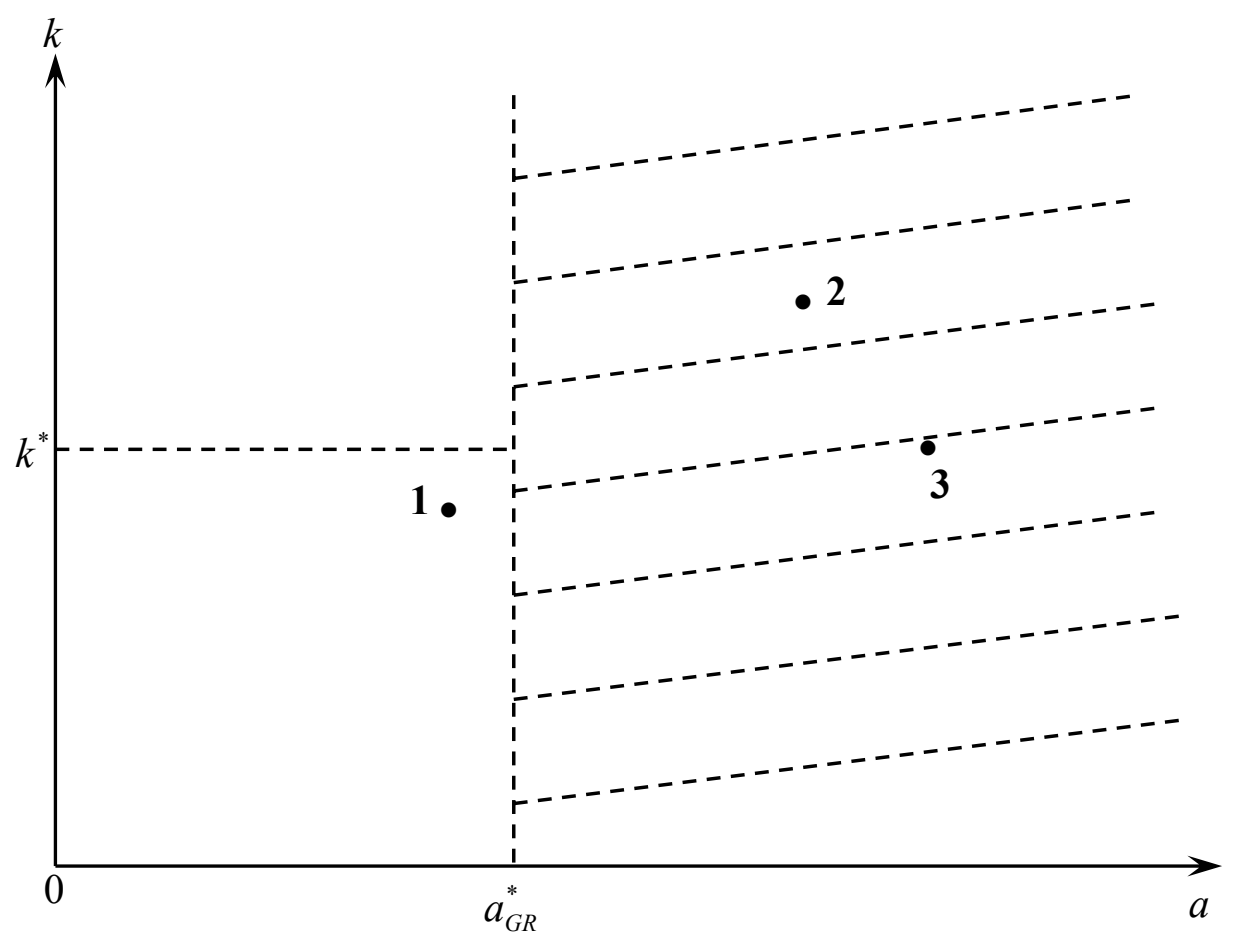

Fig. 2 A dynamically inefficient period-3 cycle. 


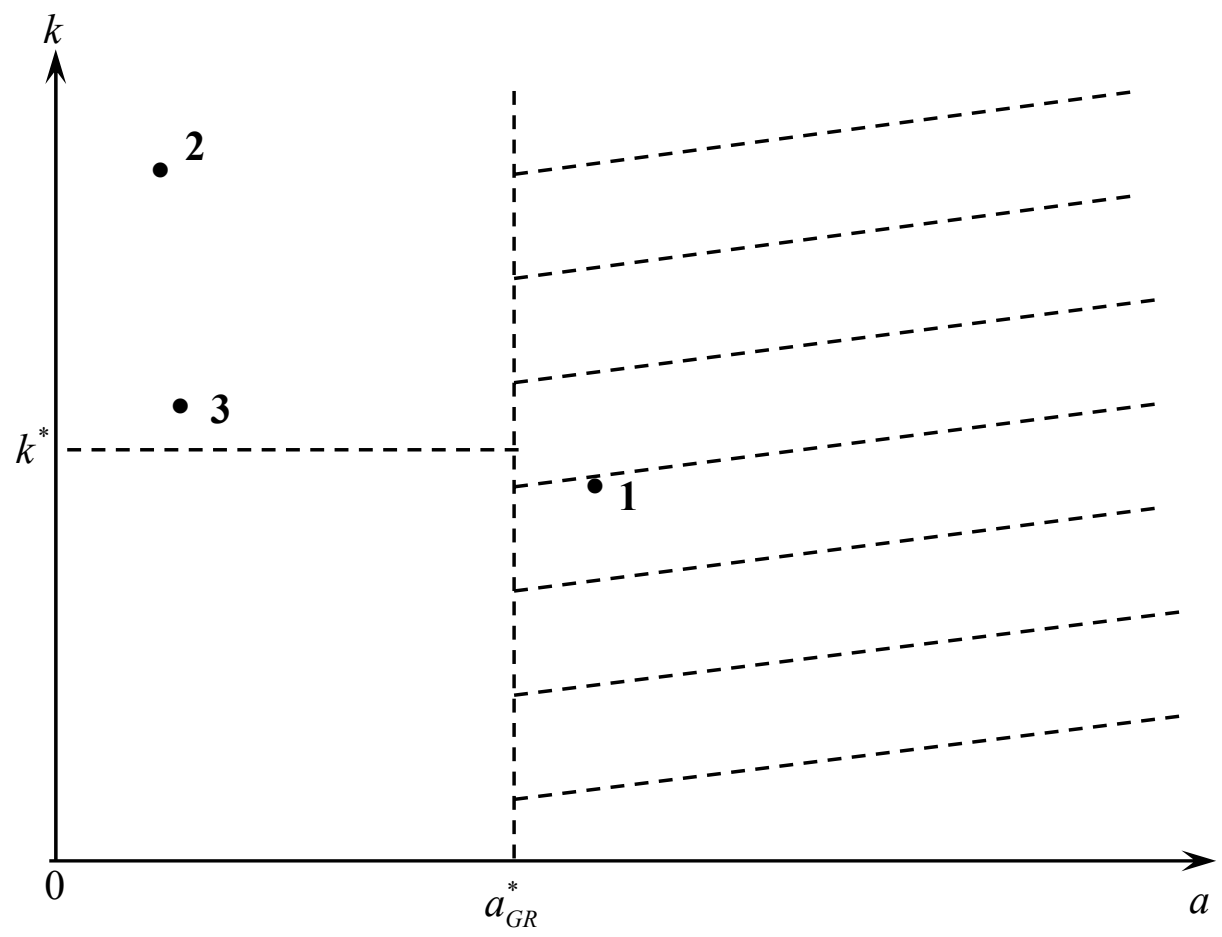

Fig. 3 A dynamically efficient period-3 cycle. 


\section{REFERENCES}

d'Aspremont, C., R. Dos Santos Ferreira, and L.-A. Gérard-Varet (1995), "Market power, coordination failures and endogenous fluctuations", H. Dixon, N. Rankin, Eds, The new macroeconomics: imperfect markets and policy effectiveness, 94-138, Cambridge: Cambridge University Press.

Azariadis, C. (1993), Intertemporal macroeconomics, Cambridge: Blackwell.

Benhabib, J., and G. Laroque (1988), "On competitive cycles in productive economies", Journal of Economic Theory 35, 145-170.

Benveniste, L. (1976), “A complete characterization of efficiency for a general capital accumulation model", Journal of Economic Theory 12, 325-37.

Cass, D. (1972), "On capital overaccumulation in the aggregative, neoclassical model of economic growth: a complete characterization", Journal of Economic Theory 4, 200-23.

Cass, D., and M.Yaari (1966), "A re-examination of the pure consumption loans model", Journal of Political Economy 74, 353-67.

Cazzavillan, G. (2001), "Indeterminacy and endogenous fluctuations with arbitrarily small externalities", Journal of Economic Theory 101, 133-57.

Cazzavillan, G., and P. Pintus (2004), "Robustness of multiple equilibria in OLG economies", Review of Economic Dynamics 7, 456-75.

Cazzavillan, G., and P. Pintus, P. (2004), "Capital externalities in OLG economies", forthcoming in Journal of Economic Dynamics and Control (temporarily published at doi:10.1016/j.jedc.2005.04.007 ).

Chattopadhyay, S., and P. Gottardi, P. (1999), "Stochastic OLG models, market structure and optimality", Journal of Economic Theory 89, 21-67. 
Diamond, P. (1965), "National debt in a neoclassical model", American Economic Review 55, 1126-1150.

Geanakoplos, J., and H. Polemarchakis (1986), "Walrasian indeterminacy and keynesian macroeconomics", Review of Economic Studies 103, 755-779.

Grandmont, J.-M. (1985), “On endogenous competitive business cycles”, Econometrica 53, 945-1045.

Grandmont, J.-M. (1993), "Expectations driven nonlinear business cycles", RheinischWestfälische Akademie des Wissenschaften 397, 7-32.

Grandmont, J.-M., and G. Laroque, G. (1993), "Stability, expectations and predetermined variables", P. Champsaur et al., Eds, Essay in honor of Edmond Malinvaud, Vol.1, Cambridge: MIT Press.

Grandmont, J.-M., P. Pintus, and R. de Vilder (1998), "Capital-labor substitution and competitive nonlinear endogenous business cycles", Journal of Economic Theory 80, 1459 .

Lloyd-Braga, T. (1995), "Increasing returns to scale and nonlinear endogenous fluctuations”, Working Paper 65, FCEE, Universidade Catolica Portuguesa.

Lloyd-Braga, T., C. Nourry, and A. Venditti (2004), "Indeterminacy in dynamic models : when Diamond meets Ramsey", GREQAM working paper series 04-52.

Malinvaud, E. (1953), "Capital accumulation and efficient allocation of resources", Econometrica 21, 233-68.

Montrucchio, L. (2004), "Cass transversality condition and sequential asset bubbles", Economic Theory 24, 645-63.

Nourry, C., A. Venditti (2006), “The overlapping generations model with endogenous labor supply: a general formulation", forthcoming in the Journal of Optimization Theory and Applications. 
Phelps, E. (1965), "Second essay on the golden rule of accumulation", American Economic Review 55, 783-814.

Pietra, T. (2004), "Sunspots, indeterminacy and Pareto inefficiency in economies with incomplete markets", Economic Theory 24, 687-99.

Pintus, P. (1997), "Indeterminacy and expectation-driven fluctuations in aggregate, general economic equilibrium models", $\mathrm{PhD}$ dissertation, EHESS, Paris, December [in French].

Reichlin, P. (1986), "Equilibrium cycles in an overlapping generations economy with production", Journal of Economic Theory 40, 89-102.

Samuelson, L. (1980), "The golden rule with endogenous labor participation rate", American Economic Review 70, 752-5.

de Vilder, R.. (1996), "Complicated endogenous business cycles under gross substitutability", Journal of Economic Theory 71, 416-442.

Woodford, M. (1984), "Indeterminacy of equilibria in the overlapping generations model: a survey", Mimeo Columbia University, May. 


\section{Dipartimento di Scienze Economiche Università Ca' Foscari Venezia Working Paper Series}

(for full list please see http://www.dse.unive.it/en/publications/)

Andrea Attar, Gwenael Piaser, Nicolàs Porteiro: 2006; A note on Common Agency models of moral hazard; Working Paper Series; 36/06

Antonio Russo, Jan van der Borg: 2006; New culture-oriented economic development trajectories: the case study of four Dutch cities; Working Paper Series; 35/06

Antonio Russo, Jan van der Borg: 2006; Towards a European Spatial Policy for Culture; Working Paper Series; 34/06

Gwenael Piaser: 2006; The Biais-Martimort-Rochet equilibrium with direct mechanisms; Working Paper Series; 33/06

Andrea Attar, Eloisa Campioni, Gwenael Piaser, Uday Rajan: 2006; On multiple agent models of moral hazard; Working Paper Series; 32/06

Cyril Hariton, Gwenael Piaser: 2006; Efficiency mitigating effects in presence of imperfect redistribution tolls; Working Paper Series; 31/06

Cyril Hariton, Gwenael Piaser: 2006; When Redistribuction Leads to Regressive Taxation; Working Paper Series; 30/06

Andrea Attar, Eloisa Campioni, Gwenael Piaser: 2006; Multiple Lending and Constrained Efficiency in the Credit Market; Working Paper Series; 29/06

Andrea Attar, Dipjyoti Majumdar, Gwenael Piaser, Nicolàs Porteiro: 2006; Common agency games with separable prferences; Working Paper Series; 28/06

Luciano Pezzolo: 2006; The rise and decline of a great power: Venice 1250-1650; Working Paper Series; 27/06

Paolo Pin: 2006; Eight Degrees of Separation; Working Paper Series; 26/06

Luciano Pezzolo, Giuseppe Tattara: 2006; Una fiera senza luogo. Was Bisenzone an offshore capital market in sixteenth-century Italy?; Working Paper Series; 25/06

Paul Cheshire, Stefano Magrini: 2006; Raising urban productivity or attracting people? Different cause, different consequences; Working Paper Series; 24/06

Paul Cheshire, Stefano Magrini: 2006; European Urban Growth: now for some problems of spaceless and weightless econometrics; Working Paper Series; 23/06

Giacomo Pasini: 2006; A Demand System with Social Interactions: Evidence from CEX; Working Paper Series; 22/06

Silvestro Di Sanzo: 2006; Output fluctuations persistence: Do cyclical shocks matter?; Working Paper Series; 21/06

Monica Billio, Silvestro Di Sanzo: 2006; Granger-causality in Markov Switching Models; Working Paper Series; 20/06

Dino Martellato: 2006; Growth and Inflation Disparities in CorridorV; Working Paper Series; 19/06

Alvaro Calzadilla, Francesco Pauli, Roberto Roson: 2006; Climate Chenge and Extreme Events: an Assessment of Economic Implications; Working Paper Series; 18/06

Roberto Roson: 2006; Equilibrium Size in Network with Indirect Network Externalities: a Comment; Working Paper Series; 17/06

Sergio Currarini: 2006; Networks Design in Games with Spillovers; Working Paper Series; $16 / 06$ 
Sergio Currarini, Francesco Feri: 2006; Delegation Versus Centralization: The Role of Externalities; Working Paper Series; 15/06

Sergio Currarini: 2006; Group Stability of Hierarchies in Games with Spillovers; Working Paper Series; 14/06

Vincenzo Rebba, Dino Rizzi: 2006; Measuring Hospitals Efficiency through Data Envelopment Analysis when Policy-makers' Preferences Matter. An Application to a sample of Italian NHS hospital; 13/06 Barbara Buchner, Carlo Carraro: 2006; Economic and enviromental effectiveness of a technology-based cliamte protocol; Working Paper Series; 12/06

Valentina Bosetti, Carlo Carraro, Marzio Galeotti: 2006; The dynamics of carbon and energy intensity in a model of endogenous technical change; Working Paper Series; $11 / 06$

Barbara Buchner, Carlo Carraro: 2006; Regional and sub-global climate blocs. A gametheoretic perspective on bottom-up climate regimes; Working Paper Series; 10/06

Carlo Carraro, Carmen Marchiori, Alessandra Sgobbi: 2006; Applications of negotiation theory to water issues; Working Paper Series; 09/06

Carlo Carraro, Carmen Marchiori, Alessandra Sgobbi: 2006; Advences in negotiation theory: bargaining, coalitions and fairness; Working Paper Series; 08/06

Barbara Buchner, Carlo Carraro: 2006; US, China and the economics of climate negotiations; Working Paper Series; 07/06

Giovanni Favero: 2006; Benetton: identifyng an image, imagining an identity; Working Paper Series; 06/06

Giovanni Favero: 2006; Old New Ceramics: Manufactures, Products and Markets in the Venetian Republic in the $17^{\text {th }}$ and $18^{\text {th }}$ Centuries; Working Paper Series; 05/06

Giovanni Favero: 2006; Le tour du monde: les mémories de voyage d'un émigrant de Schio en Russie et au Canada au début du $\mathrm{XX}^{\mathrm{e}}$ siécle; Working Paper Series; $04 / 06$

Paola Lanaro, Giovanni Favero: 2006; Teaching urban history in Italian Universities; Working Paper Series; 03/06

Roberto Roson: 2006; Introduction imperfect competition in CGE models: technical aspects and implications; Working Paper Series; 02/06

Ignazio Musu: 2006; Intellectual property rights and biotechnology: how to improve the present patent system; Working Paper Series; 01/06 